\title{
Antibiotic-induced depletion of gut microbiota increases systemic exposure of clopidogrel active metabolite in type 2 diabetic rats
}

\author{
Xue Chen ${ }^{1}$, Yingrui Liu ${ }^{1}$, Hongwei Yao ${ }^{1}$, Wenfang Song ${ }^{1}$, Meng Pan ${ }^{1}$, Yu Song ${ }^{2}$, Jingkai \\ $\mathrm{Gu}^{1}$, and Yingjie $\mathrm{Guo}^{1}$ \\ ${ }^{1}$ Jilin University \\ ${ }^{2}$ Hainan Tropical Ocean University
}

November 26, 2021

\begin{abstract}
Background and Purpose: The current study investigated whether the manipulation of gut microbiome through treatment with an antibiotic cocktail can alter the bioavailability of clopidogrel active metabolite (Clop-AM) in T2DM rats. Experimental Approach: Control and T2DM rats were orally administered with either vehicle or an antibiotic cocktail containing ampicillin, neomycin, metronidazole, and vancomycin for 5 consecutive days. The levels of clopidogrel (Clop) and its metabolites were measured by LC-MS/MS. Biochemical parameters, liver microsome metabolism, mRNA, protein or activity of Clop- metabolizing enzymes and transporter, and 16S rRNA sequence of fecal samples were analyzed to explain any altered pharmacokinetic profile of Clop-AM. Key Results: Antibiotic administration markedly alleviated T2DM rats' phenotypes including hyperglycemia, hyperlipidemia, insulin resistance, liver dysfunction and inflammation. Meanwhile, the reduced systemic exposure of Clop-AM in T2DM rats as compared to control rats was significantly reversed after antibiotic treatment, accompanied with the decreased expression of P-glycoprotein (P-gp) in small intestine, suggesting P-gp-based Clop absorption might be promoted, consequently making more Clop available for Clop-AM formation. Interestingly, fecal microbiome analysis exhibited the reduced microbial amount and the altered microbial composition in antibiotic-treated T2DM rats. Especially, there was an inconsistent change of P-gp levels between T2DM rats and SW480 cells after antibiotic treatment, suggesting antibiotic-induced microbiome depletion, not the direct role of antibiotics is associated with the enhanced Clop-AM plasma exposure in T2DM rats. Conclusion and Implication: The findings show that gut microbiota modulation is an effective therapeutic strategy to enhance Clop-AM generation under T2DM conditions.
\end{abstract}

\section{Hosted file}

Manuscript.docx available at https://authorea.com/users/448137/articles/546999-antibioticinduced-depletion-of-gut-microbiota-increases-systemic-exposure-of-clopidogrel-activemetabolite-in-type-2-diabetic-rats 

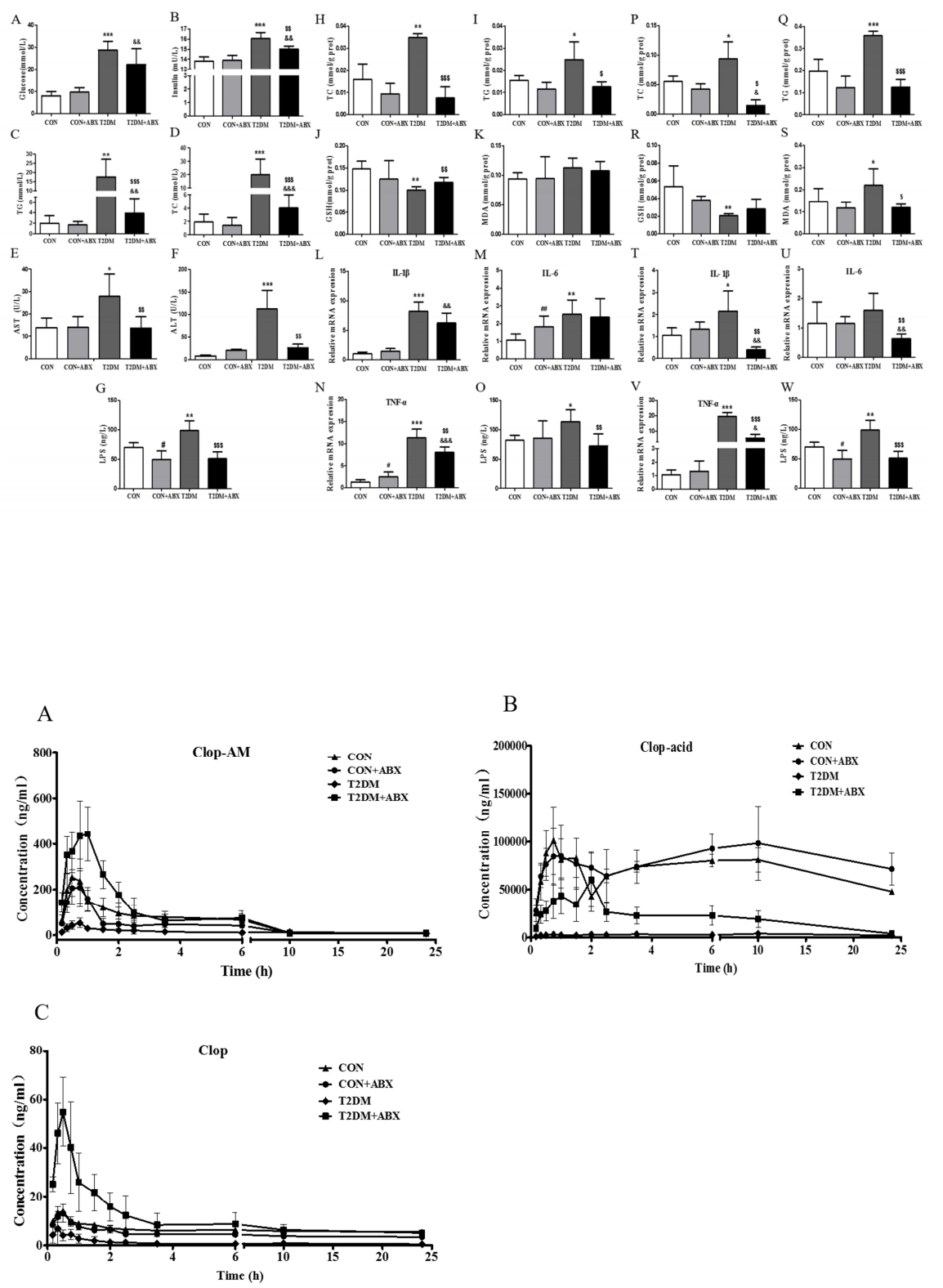

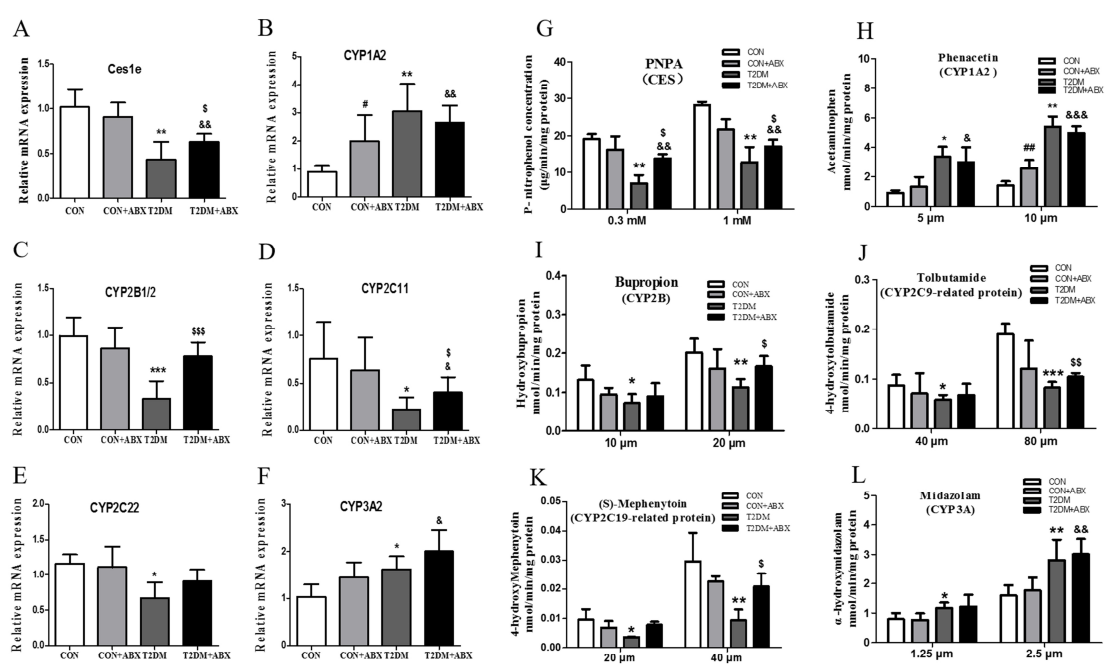

K
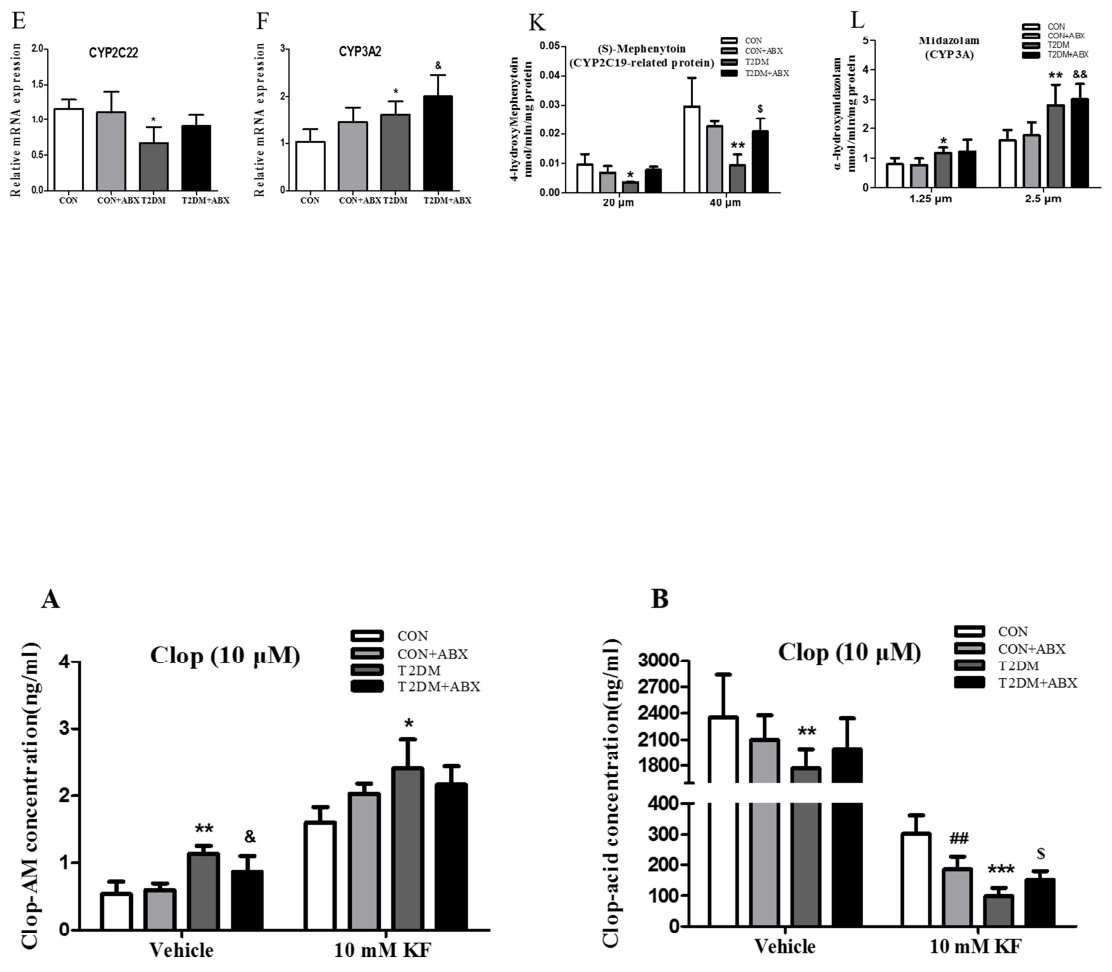


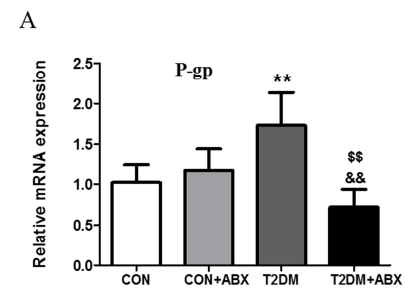

C
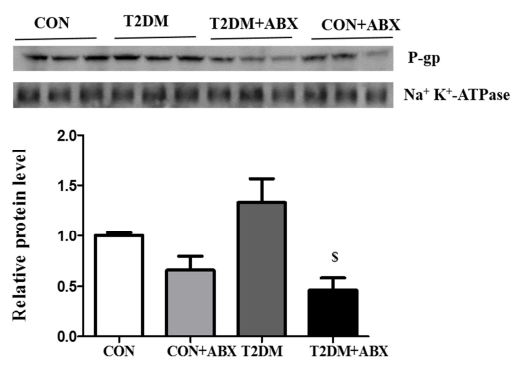

B
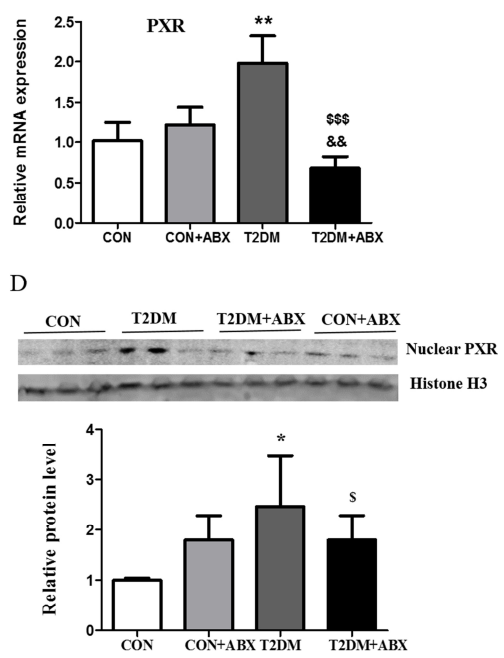
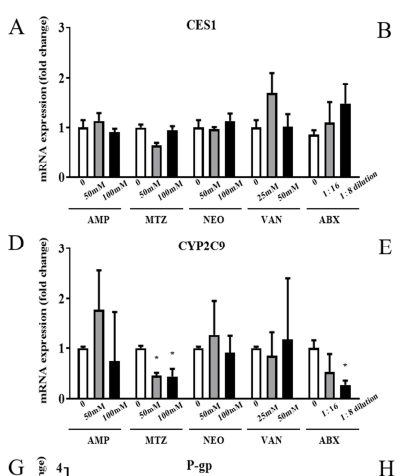

CYP1A2
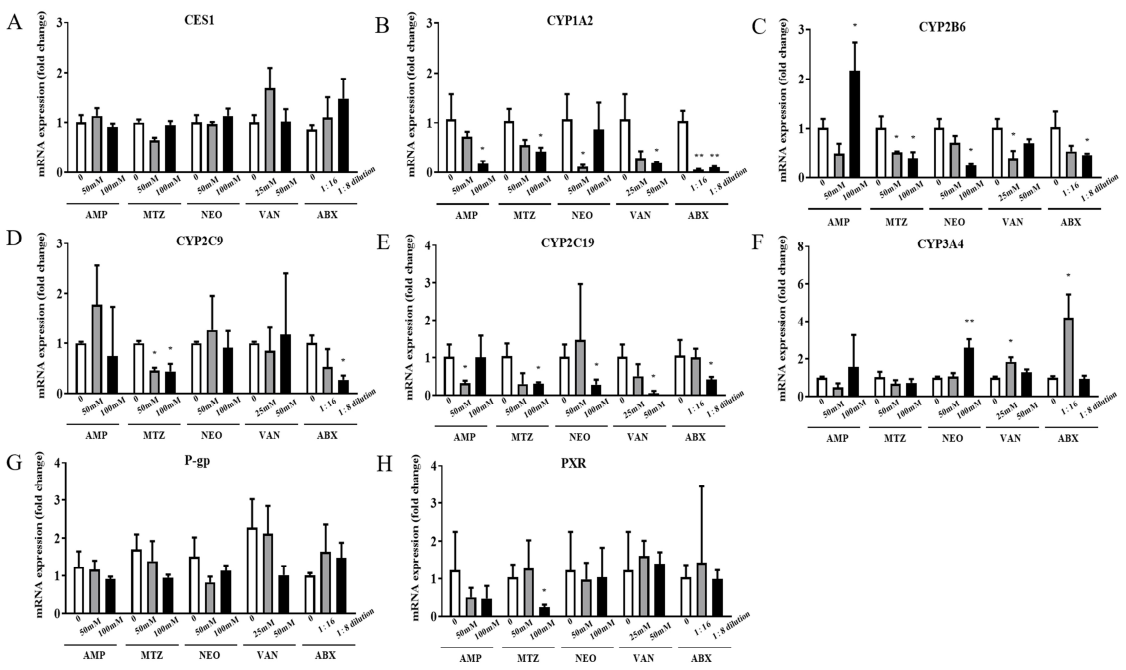

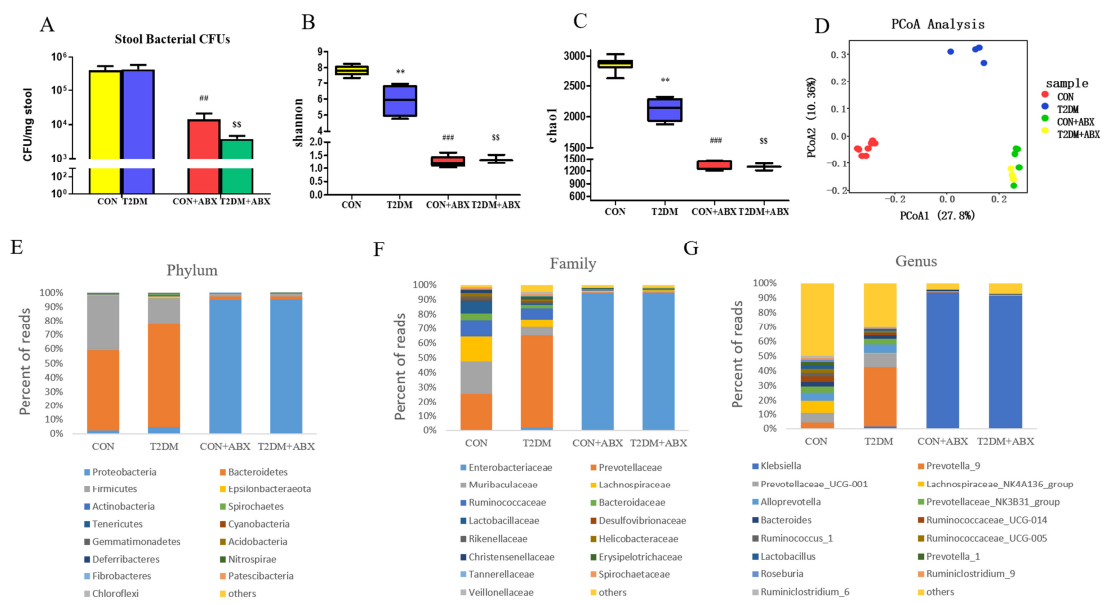

A

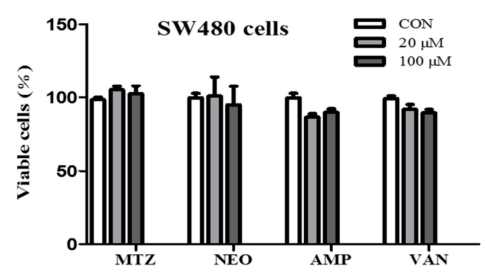

$\mathrm{C}$

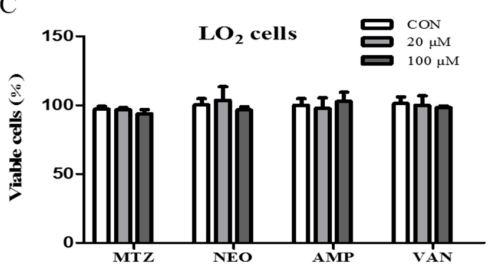

B

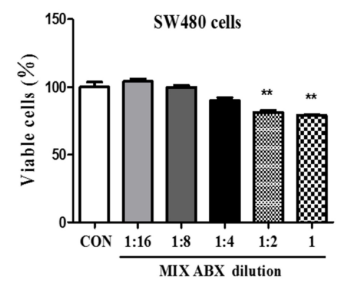

$\mathrm{D}$

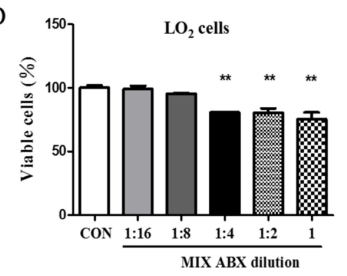

\section{Hosted file}

Tables.docx available at https://authorea.com/users/448137/articles/546999-antibioticinduced-depletion-of-gut-microbiota-increases-systemic-exposure-of-clopidogrel-activemetabolite-in-type-2-diabetic-rats 